Research Article

\title{
Error Modeling and Analysis for InSAR Spatial Baseline Determination of Satellite Formation Flying
}

\author{
Jia Tu, Defeng Gu, Yi Wu, and Dongyun Yi \\ Department of Mathematics and Systems Science, College of Science, \\ National University of Defense Technology, Changsha 410073, China \\ Correspondence should be addressed to Jia Tu, tu_jia_jia@yahoo.com.cn
}

Received 30 September 2011; Revised 9 December 2011; Accepted 12 December 2011

Academic Editor: Silvia Maria Giuliatti Winter

Copyright (C) 2012 Jia Tu et al. This is an open access article distributed under the Creative Commons Attribution License, which permits unrestricted use, distribution, and reproduction in any medium, provided the original work is properly cited.

\begin{abstract}
Spatial baseline determination is a key technology for interferometric synthetic aperture radar (InSAR) missions. Based on the intersatellite baseline measurement using dual-frequency GPS, errors induced by InSAR spatial baseline measurement are studied in detail. The classifications and characters of errors are analyzed, and models for errors are set up. The simulations of single factor and total error sources are selected to evaluate the impacts of errors on spatial baseline measurement. Single factor simulations are used to analyze the impact of the error of a single type, while total error sources simulations are used to analyze the impacts of error sources induced by GPS measurement, baseline transformation, and the entire spatial baseline measurement, respectively. Simulation results show that errors related to GPS measurement are the main error sources for the spatial baseline determination, and carrier phase noise of GPS observation and fixing error of GPS receiver antenna are main factors of errors related to GPS measurement. In addition, according to the error values listed in this paper, $1 \mathrm{~mm}$ level InSAR spatial baseline determination should be realized.
\end{abstract}

\section{Introduction}

Close formation flying satellites equipped with synthetic aperture radar (SAR) antenna could provide advanced science opportunities, such as generating highly accurate digital elevation models (DEMs) from Interferometric SAR (InSAR) [1, 2]. Compared to a single SAR satellite system, the performance of two SAR satellites flying in close formation can be greatly enhanced. Nowadays, close satellite formation flying has become the focus of space technology and geodetic surveying.

In order to realize the advanced space mission goal of InSAR mission, the highprecision determination of inter-satellite interferometric baseline [3] is a fundamental issue. 
Take the TanDEM-X mission for instance. TanDEM-X mission is the first bistatic single-pass SAR satellite formation, which is formed by adding a second TanDEM- $X$, almost identical spacecraft, to TerraSAR-X and flying the two satellites in a closely controlled formation. The primary mission goal is the derivation of a high-precision global DEM according to highresolution terrain information (HRTI) level 3 accuracy [4-6]. The generation of accurate InSAR-derived DEMs requires a precise knowledge of the interferometric baseline with an accuracy of $1 \mathrm{~mm}$ (1D, RMS) [7]. Therefore high-precision determination of inter-satellite interferometric baseline is a prerequisite for InSAR mission.

The interferometric baseline is defined as the separation between two SAR antennas that receive echoes of the same ground area [8]. Based on this definition, the interferometric baseline can be denoted as the resultant vector of temporal baseline and spatial baseline, that is,

$$
\mathbf{S}_{2}\left(t_{2}\right)-\mathbf{S}_{1}\left(t_{1}\right)=\mathbf{S}_{2}\left(t_{2}\right)-\mathbf{S}_{1}\left(t_{2}\right)+\mathbf{S}_{1}\left(t_{2}\right)-\mathbf{S}_{1}\left(t_{1}\right)
$$

where $t_{1}, t_{2}$ are epochs that two SAR antennas receive echoes of the same ground area, $\mathbf{S}_{1}(t)$, $\mathbf{S}_{2}(t)$ represent the positions of SAR antenna phase centers of satellite 1 and satellite 2 at epoch $t$ in International Terrestrial Reference Frame (ITRF), respectively, $\mathbf{S}_{2}\left(t_{2}\right)-\mathbf{S}_{1}\left(t_{2}\right)$ is the spatial baseline, $\mathbf{S}_{1}\left(t_{2}\right)-\mathbf{S}_{1}\left(t_{1}\right)$ is the temporal baseline which is the velocity integral of satellite 1 . For close formation flying $(1 \mathrm{~km}-2 \mathrm{~km})$ with single-pass bistatic acquisitions, the deviation of epochs that two SAR antennas receive echoes of the same ground area is typically on the millisecond level. When the velocity is determined on the $\mathrm{mm} / \mathrm{s}$ level, its influence in the temporal baseline can be neglected. Therefore, the accuracy of interferometric baseline is mainly determined by the accuracy of spatial baseline. Note that only spatial baseline is considered in this paper.

The spaceborne dual-frequency GPS measurement scheme [9-11] is widely used for inter-satellite baseline determination currently. This scheme for spatial baseline determination consists of two steps. Firstly, the relative position of two formation satellites is determined by dual-frequency GPS measurement, and then spatial baseline is transformed from inter-satellite relative position. The relative position here is the vector that links the mass centers of two formation satellites.

In our research, impacts of the errors introduced by spatial baseline measurement are analyzed. This paper starts with a description of spatial baseline measurement using dualfrequency GPS. The baseline transformation from the relative position to spatial baseline is given. In a second step, errors are classified into two groups: errors related to GPS measurement and errors related to baseline transformation. The error characters are studied, and the impact of each error on spatial baseline determination is analyzed from theoretical aspect. Then the impacts of each error and total errors on spatial baseline determination are analyzed by single factor simulations and total error sources simulations. At last, conclusions are shown.

\section{Generation of Spatial Baseline}

In preparation for latter description some coordinate systems are introduced at first, which are illustrated in Figure 1. Coordinate systems employed in this paper contain Conventional Inertial Reference Frame (CIRF), ITRF, satellite body coordinate system, and satellite orbit 


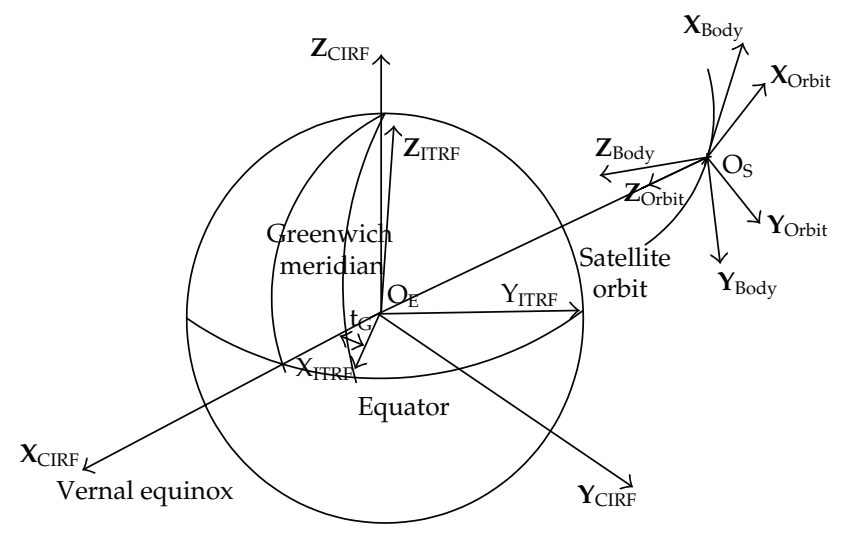

Figure 1: Definitions of coordinate systems employed in this paper. CIRF, ITRF, satellite body coordinate system, and satellite orbit coordinate system are denoted as $\mathbf{O}_{\mathrm{E}}-\mathbf{X}_{\mathrm{CIRF}} \mathbf{Y}_{\mathrm{CIRF}} \mathbf{Z}_{\mathrm{CIRF}}, \mathbf{O}_{\mathrm{E}}-\mathbf{X}_{\text {ITRF }} \mathbf{Y}_{\text {ITRF }} \mathbf{Z}_{\text {ITRF }}, \mathbf{O}_{S^{-}}$ $\mathbf{X}_{\text {Body }} \mathbf{Y}_{\text {Body }} \mathbf{Z}_{\text {Body }}$, and $\mathbf{O}_{\mathrm{S}}-\mathbf{X}_{\text {Orbit }} \mathbf{Y}_{\text {Orbit }} \mathbf{Z}_{\text {Orbit }}$, respectively. $\mathbf{O}_{\mathrm{E}}$ is the geocenter, and $\mathbf{O}_{\mathrm{S}}$ is the mass center of satellite.

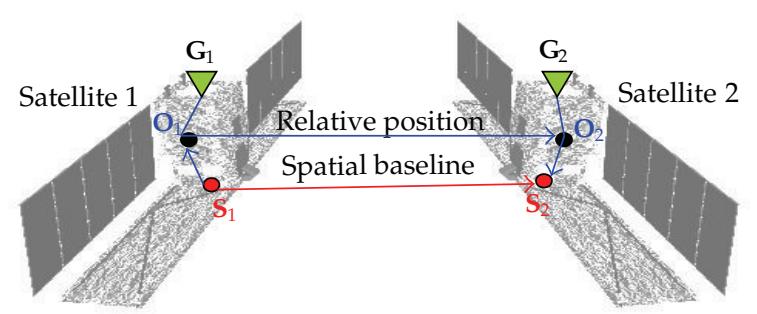

Figure 2: Geometric relation for spatial baseline determination. $\mathbf{G}_{1}$ and $\mathbf{G}_{2}$ are GPS receiver antenna phase centers, $\mathbf{O}_{1}$ and $\mathbf{O}_{2}$ are mass centers, and $\mathbf{S}_{1}$ and $\mathbf{S}_{2}$ are SAR antenna phase centers.

coordinate system. CIRF used here is J2000.0 inertial system and ITRF is ITRF2000 system. The definitions of these coordinate systems can be found in [12].

As the spatial baseline is determined by spaceborne dual-frequency GPS measurement scheme, the entire process of spatial baseline determination consists of relative positioning and baseline transformation. Figure 2 is the geometric relation for spatial baseline determination.

Relative positioning is the determination of $\mathbf{O}_{1} \mathbf{O}_{2}$ by dual-frequency GPS observation data. As the real position of signal reception is the phase center $\mathbf{G}_{i}(i=1,2)$ of GPS receiver antenna, GPS observation data has to be revised to the mass center $\mathbf{O}_{i}(i=1,2)$ of satellite using the phase center data of GPS receiver antenna during relative positioning.

From Figure 2, baseline transformation can be described as follows:

$$
\mathbf{S}_{1} \mathbf{S}_{2}=\mathbf{O}_{1} \mathbf{O}_{2}+\mathbf{M}_{1} \cdot \mathbf{S}_{1} \mathbf{O}_{1}-\mathbf{M}_{2} \cdot \mathbf{S}_{2} \mathbf{O}_{2}
$$

where $\mathbf{S}_{1} \mathbf{S}_{2}$ is the spatial baseline in ITRF, $\mathbf{O}_{1} \mathbf{O}_{2}$ is the relative position of two satellites in ITRF, $\mathbf{S}_{i} \mathbf{O}_{i}(i=1,2)$ is a vector that links SAR antenna phase center to mass center of satellite in body coordinate system of Satellite $i, \mathbf{M}_{i}(i=1,2)$ is a transformation matrix of Satellite $i$ from satellite body coordinate system to ITRF. The flow chart of spatial baseline determination is shown in Figure 3. 


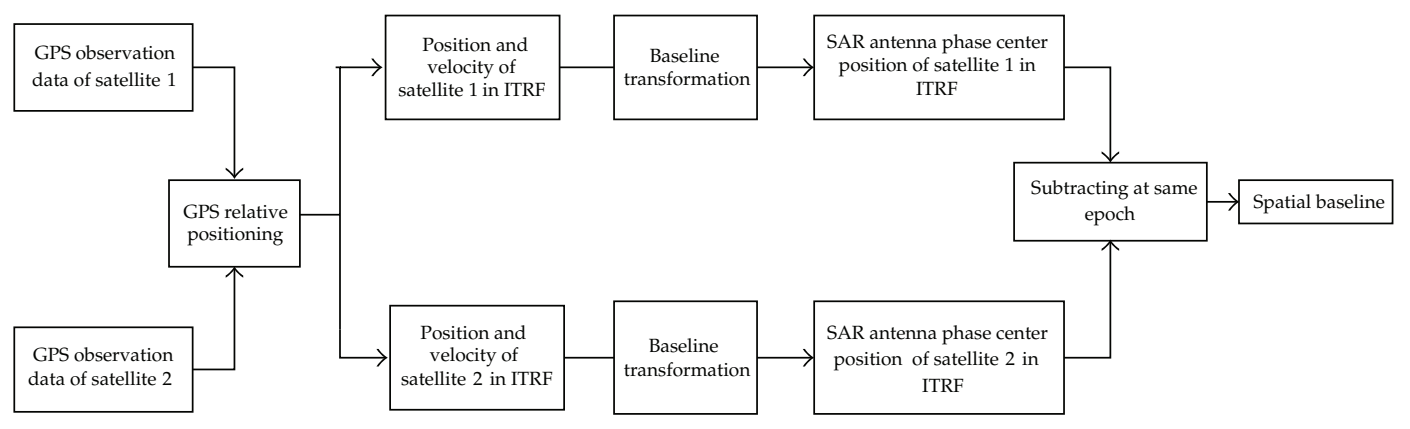

Figure 3: Flow chat of spatial baseline determination.

\section{Errors of Spatial Baseline Measurement}

According to the generation of spatial baseline in Section 2, the errors of spatial baseline measurement can be classified into two groups: errors related to GPS measurement, which are introduced by relative positioning using dual-frequency GPS measurement, and errors related to baseline transformation, which are generated by the transformation from relative position to spatial baseline.

\subsection{Errors Related to GPS Measurement}

The relative positions of two satellites are determined by the reduced dynamic carrier phase differential GPS approach. In this approach, the absolute orbits of one reference satellite (Satellite 1) are fixed, which are determined by the zero-difference reduced dynamic batch least squares approach based on GPS measurements of single satellite. Only the relative positions are estimated by reduced dynamic batch least-squares approach based on differential GPS measurements. The integer double difference ambiguities for relative positioning are resolved by estimating wide-lane and narrow-lane combinations [13]. The well-known Least-Squares Ambiguity Decorrelation Adjustment (LAMBDA) method [14, $15]$ is implemented for the integer estimate.

By differenced GPS observation, common errors can be eliminated or reduced. International GNSS Service (IGS) final GPS ephemeris product (orbit product and clock product) [16] is often adopted for orbit determination based on GPS observation. The accuracy of GPS final orbit product is presently on the order of $2.5 \mathrm{~cm}$. For $2 \mathrm{~km}$ separation of satellite formation, the impact of GPS ephemeris error on single-difference GPS observation is about $0.0025 \mathrm{~mm}$ [17], which can be neglected. The impact of GPS clock error can be well cancelled out by differential GPS observation. Due to the close separation $(1 \mathrm{~km}-$ $2 \mathrm{~km}$ ) and similar materials, configuration, and in-flight environment of formation satellites, near-field multipath, thermal distortions of satellites, and other external perturbations can also be effectively reduced by differential GPS observation. In addition, the influence of differential ionospheric path delay is mainly from the first order, which can be eliminated by constructing ionosphere free differential GPS observation. Therefore, the errors related to GPS measurement that have to be considered consist of noise of GPS carrier phase measurement, ground calibration error of GPS receiver antenna phase center, error of satellite attitude measurement, and fixing error of GPS receiver antenna. 


\subsubsection{Noise of GPS Carrier Phase Measurement}

The quality of GPS carrier phase observation data used is of utmost importance for relative positioning. The noise of GPS carrier phase measurement belongs to random error, which cannot directly be eliminated by GPS differential observation. Take the BlackJack receiver and its commercial Integrated GPS and Occultation Receiver (IGOR) version, for example, which are widely used for geodetic grade space missions and exhibit a representative noise level of $1 \mathrm{~mm}$ for $L 1$ and $L 2$ carrier phase measurements [18]. The reduced dynamic relative positioning approach makes use of dynamical models of the spacecraft motion to constrain the resulting relative position estimates, which allows an averaging of measurements from different epochs. The influence of GPS carrier phase noise can be effectively reduced by reduced dynamic relative positioning approach.

\subsubsection{Ground Calibration Error of GPS Receiver Antenna Phase Center}

The phase center location accuracy of the GPS receiver antenna will directly affect the veracity of GPS observation modeling. GPS receiver antenna phase center is the instantaneous location of the GPS receiver antenna where the GPS signal is actually received. It depends on intensity, frequency, azimuth, and elevation of GPS receiving signal.

The phase center locations can be described by the mechanical antenna reference point $(\mathrm{ARP})$, a phase center offset (PCO) vector, and phase center variations (PCVs). The PCO vector describes the difference between the mean center of the wave front and the ARP. PCVs represent direction-dependent distortions of the wave front, which can be modeled as a consistent function that depends on azimuth and elevation of the observation from the position indicated by the PCO vector. The position of GPS receiver antenna phase center can be measured by ground calibration, such as using an anechoic chamber and using field calibration techniques [18, 19]. Take the SEN67-1575-14+CRG antenna system for instance. It is a dual-frequency GPS receiver antenna and has been used for TanDEM$X$ mission. Its phase center has been measured by automated absolute field calibration [20]. The mean value of calibration result is shown in Figure 4 that the pattern of PCVs has obvious character of systematic deviation. The maximum value for the mean PCVs on ionospherefree combination can reach to $1.5 \mathrm{~cm}$. In addition, there also exist random errors in the same direction of different receptions. The random errors are similar to the noise of GPS carrier phase measurement and can also be smoothed by reduced dynamic relative positioning approach.

As there is a slim difference between the line of sight (LOS) vectors of two satellites during close formation flying, the common systematic errors of GPS receiver antenna phase center and near-field multipath can be eliminated by differential GPS observation. Therefore, the same type of GPS receiver antenna has to be selected for both formation satellites in order to reduce the impact of these errors.

\subsubsection{Error of Satellite Attitude Measurement}

Satellite attitude data are obtained from star camera observations and provided as quaternion. The error of satellite attitude measurement consists of a slowly varying bias and a random error. Its impact on GPS relative positioning appears on the correction for GPS observation data of single satellite, that is, the reference point of GPS observation data has to 


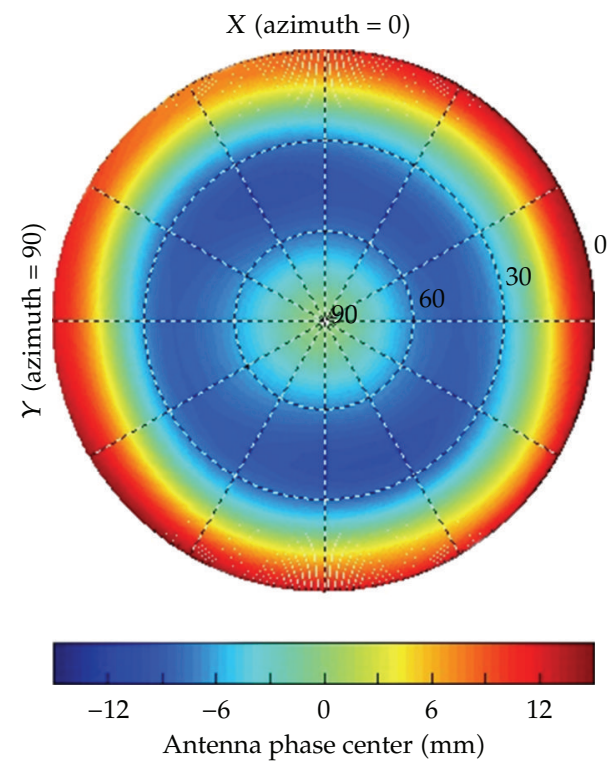

Figure 4: Ground calibrated mean PCVs result of SEN67-1575-14+CRG antenna on ionosphere-free combination.

be corrected from GPS receiver antenna phase center to the mass center of satellite by satellite attitude data and GPS receiver antenna phase center data. Take Satellite 1 for instance. The correction in direction of LOS vector $\mathbf{e}_{1}^{j}$ (in CIRF) is given by

$$
\begin{aligned}
& \delta_{\text {Offset }, 1}=-\left(\mathbf{e}_{1}^{j}\right)^{\mathrm{T}} \cdot \mathbf{M}_{\text {Body_CIRF }} \cdot \mathbf{O}_{1} \mathbf{G}_{1} \\
& \mathbf{M}_{\text {Body_CIRF }}=\mathbf{M}_{\text {Orbit_CIRF }} \cdot \mathbf{M}_{\text {Body_Orbit }}
\end{aligned}
$$

where $\mathbf{O}_{1} \mathbf{G}_{1}$ is GPS receiver antenna phase center location in body coordinate system of Satellite 1, $\mathbf{M}_{\text {Body_CIRF }}$ is the transformation matrix from body coordinate system of Satellite 1 to CIRF and can be obtained by attitude quaternion data, $\mathbf{M}_{\text {Body_Orbit }}$ is the transformation matrix from body coordinate system to orbit coordinate system of Satellite 1, and $\mathbf{M}_{\text {Orbit_CIRF }}$ is the transformation matrix from orbit coordinate system of Satellite 1 to CIRF.

Assuming that the Euler angles are $\varphi, \theta$, and $\psi$ respectively, we can get

$$
\mathbf{M}_{\text {Body_Orbit }}=\mathbf{R}_{X}(\varphi) \cdot \mathbf{R}_{Y}(\theta) \cdot \mathbf{R}_{Z}(\psi),
$$

where $\mathbf{R}_{X}(\varphi), \mathbf{R}_{Y}(\theta), \mathbf{R}_{Z}(\psi)$ are rotation matrices around roll axis, pitch axis, and yaw axis, respectively.

Assuming that the errors of Euler angle measurements are $\varepsilon_{\varphi}, \varepsilon_{\theta}$, and $\varepsilon_{\psi}$, respectively, and the corresponding error matrix of $\mathbf{M}_{\text {Body_CIRF }}$ is $\varepsilon_{\mathbf{M}}$, the relation between $\varepsilon_{\mathbf{M}}$ and $\varepsilon_{\varphi}, \varepsilon_{\theta}, \varepsilon_{\psi}$ can be expressed as

$$
\varepsilon_{\mathbf{M}}=\mathbf{M}_{\text {Orbit_CIRF }} \cdot\left(\frac{\partial \mathbf{R}_{X}}{\partial \varphi} \mathbf{R}_{Y} \mathbf{R}_{Z} \cdot \varepsilon_{\varphi}+\mathbf{R}_{X} \frac{\partial \mathbf{R}_{Y}}{\partial \theta} \mathbf{R}_{Z} \cdot \varepsilon_{\theta}+\mathbf{R}_{X} \mathbf{R}_{Y} \frac{\partial \mathbf{R}_{Z}}{\partial \psi} \cdot \varepsilon_{\psi}\right) .
$$


Furthermore, the impact of Euler angle errors on $\mathbf{M}_{\text {Body_CIRF }} \cdot \mathbf{O}_{1} \mathbf{G}_{1}$ in (3.1) can be obtained as

$$
\begin{aligned}
\varepsilon_{\mathbf{M}_{\text {Body_CIRF }} \cdot \mathbf{O}_{1} \mathbf{G}_{1}}= & \mathbf{M}_{\text {Orbit_CIRF }} \\
& \cdot\left(\frac{\partial \mathbf{R}_{X}}{\partial \varphi} \mathbf{R}_{Y} \mathbf{R}_{Z} \cdot \mathbf{O}_{1} \mathbf{G}_{1} \cdot \varepsilon_{\varphi}+\mathbf{R}_{X} \frac{\partial \mathbf{R}_{Y}}{\partial \theta} \mathbf{R}_{Z} \cdot \mathbf{O}_{1} \mathbf{G}_{1} \cdot \varepsilon_{\theta}+\mathbf{R}_{X} \mathbf{R}_{Y} \frac{\partial \mathbf{R}_{Z}}{\partial \psi} \cdot \mathbf{O}_{1} \mathbf{G}_{1} \cdot \varepsilon_{\psi}\right) .
\end{aligned}
$$

$\varepsilon_{\mathbf{M}_{\text {Body_CIRF }} \cdot \mathbf{O}_{1} \mathbf{G}_{1}}$ is a three-dimensional random vector and its magnitude can be described as the mean value of space radius, that is

$$
\boldsymbol{\sigma}_{\mathrm{M}_{\text {Body_CIRF }} \cdot \mathbf{O}_{1} \mathbf{G}_{1}}^{2}=\mathbf{E}\left(\left|\varepsilon_{\mathbf{M}_{\text {Body }- \text { IIRF }} \cdot \mathbf{O}_{1} \mathbf{G}_{1}}\right|^{2}\right)=\mathbf{E}\left(\varepsilon_{\mathbf{M}_{\text {Body-CIRF }}^{T} \mathbf{O}_{1} \mathbf{G}_{1}}^{T} \cdot \varepsilon_{\mathbf{M}_{\text {Body_CIRF }} \cdot \mathbf{O}_{1} \mathbf{G}_{1}}\right)
$$

where $|\cdot|$ denotes the magnitude of a vector, $\mathrm{E}(\cdot)$ denotes the expectation of a random variable. Assuming Euler angle errors of different axes are independent, we can get

$$
\begin{aligned}
\boldsymbol{\sigma}_{\mathbf{M}_{\text {Body_CIRF }}^{2} \mathbf{O}_{1} \mathbf{G}_{1}}= & \left|\mathbf{M}_{\text {Orbit_CIRF }} \cdot \frac{\partial \mathbf{R}_{X}}{\partial \varphi} \mathbf{R}_{Y} \mathbf{R}_{Z} \cdot \mathbf{O}_{1} \mathbf{G}_{1}\right|^{2} \cdot\left(\operatorname{Var}\left(\varepsilon_{\varphi}\right)+\left(\mathbf{E}\left(\varepsilon_{\varphi}\right)\right)^{2}\right) \\
& +\left|\mathbf{M}_{\text {Orbit_CIRF }} \cdot \mathbf{R}_{X} \frac{\partial \mathbf{R}_{Y}}{\partial \theta} \mathbf{R}_{Z} \cdot \mathbf{O}_{1} \mathbf{G}_{1}\right|^{2} \cdot\left(\operatorname{Var}\left(\varepsilon_{\theta}\right)+\left(\mathbf{E}\left(\varepsilon_{\theta}\right)\right)^{2}\right) \\
& +\left|\mathbf{M}_{\text {Orbit_CIRF }} \cdot \mathbf{R}_{X} \mathbf{R}_{Y} \frac{\partial \mathbf{R}_{Z}}{\partial \psi} \cdot \mathbf{O}_{1} \mathbf{G}_{1}\right|^{2} \cdot\left(\operatorname{Var}\left(\varepsilon_{\psi}\right)+\left(\mathbf{E}\left(\varepsilon_{\psi}\right)\right)^{2}\right),
\end{aligned}
$$

where $\operatorname{Var}(\cdot)$ denotes the variation of a random variable.

As $\mathbf{R}_{X}(\varphi), \mathbf{R}_{Y}(\theta), \mathbf{R}_{Z}(\psi)$, and $\mathbf{M}_{\text {Orbit_CIRF }}$ are orthogonal matrices, for any $\mathbf{v} \in \mathbf{R}^{3}$, we can get

$$
\begin{gathered}
\left|\mathbf{R}_{X} \cdot \mathbf{v}\right|^{2}=|\mathbf{v}|^{2}, \quad\left|\frac{\partial \mathbf{R}_{X}}{\partial \varphi} \cdot \mathbf{v}\right|^{2} \leq|\mathbf{v}|^{2} \\
\left|\mathbf{R}_{Y} \cdot \mathbf{v}\right|^{2}=|\mathbf{v}|^{2}, \quad \\
\left|\frac{\partial \mathbf{R}_{Y}}{\partial \theta} \cdot \mathbf{v}\right|^{2} \leq|\mathbf{v}|^{2} \\
\left|\mathbf{R}_{Z} \cdot \mathbf{v}\right|^{2}=|\mathbf{v}|^{2}, \quad\left|\frac{\partial \mathbf{R}_{Z}}{\partial \psi} \cdot \mathbf{v}\right|^{2} \leq|\mathbf{v}|^{2} \\
\left|\mathbf{M}_{\text {Orbit_CIRF }} \cdot \mathbf{v}\right|^{2}=|\mathbf{v}|^{2} .
\end{gathered}
$$

Taking (3.7) into (3.6), we can get

$$
\boldsymbol{\sigma}_{\mathbf{M}_{\text {Body_CRF }} \cdot \mathbf{O}_{1} \mathbf{G}_{1}}^{2} \leq\left|\mathbf{O}_{1} \mathbf{G}_{1}\right|^{2} \cdot\left[\operatorname{Var}\left(\varepsilon_{\varphi}\right)+\left(\mathbf{E}\left(\varepsilon_{\varphi}\right)\right)^{2}+\operatorname{Var}\left(\varepsilon_{\theta}\right)+\left(\mathbf{E}\left(\varepsilon_{\theta}\right)\right)^{2}+\operatorname{Var}\left(\varepsilon_{\psi}\right)+\left(\mathbf{E}\left(\varepsilon_{\psi}\right)\right)^{2}\right] .
$$


Hence,

$$
\sigma_{\delta_{\text {Offset }, 1}^{2}}^{2}=\mathbf{E}\left(\left|\varepsilon_{\delta_{\text {Offset }}}\right|^{2}\right)=\mathbf{E}\left(\left|\left(\mathbf{e}_{1}^{j}\right)^{\mathrm{T}} \cdot \boldsymbol{\varepsilon}_{\mathbf{M}_{\text {Body_CIRF }} \cdot \mathbf{O}_{1} \mathbf{G}_{1}}\right|^{2}\right) \leq \mathrm{E}\left(\left|\varepsilon_{\mathbf{M}_{\text {Body_CIRF }} \cdot \mathbf{O}_{1} \mathbf{G}_{1}}\right|^{2}\right) .
$$

For differential GPS observation, the impact of attitude determination error on two satellites can be given as follows

$$
\sigma_{\Delta \delta_{\text {Offset }}} \leq \sqrt{\sigma_{\delta_{\text {Offset }, 1}^{2}}^{2}+\sigma_{\delta_{\text {Offset }, 2}^{2}}^{2}}
$$

According to the TanDEM-X missions, the attitude determination accuracy has a slowly varying bias of $\pm 0.005^{\circ}$ in the yaw, pitch, and roll components plus a $0.003^{\circ}$ sigma random error [21]. From (3.8), (3.9), and (3.10), we can get

$$
\sigma_{\Delta \delta_{\text {Offset }}} \leq \sqrt{3 \cdot\left(\left|\mathbf{O}_{1} \mathbf{G}_{1}\right|^{2}+\left|\mathbf{O}_{2} \mathbf{G}_{2}\right|^{2}\right) \cdot\left[\left(\frac{0.005}{180} \pi\right)^{2}+\left(\frac{0.003}{180} \pi\right)^{2}\right]} .
$$

Take the GPS receiver antenna ARP location of TanDEM-X mission for instance, that is,

$$
\left|\mathbf{O}_{1} \mathbf{G}_{1}\right|=\left|\mathbf{O}_{2} \mathbf{G}_{2}\right|=1.8976 \mathrm{~m},
$$

we can get

$$
\sigma_{\Delta \delta_{\text {Offset }}} \leq 0.47 \mathrm{~mm} \text {. }
$$

\subsubsection{Fixing Error of GPS Receiver Antenna}

The fixing error of GPS receiver antenna is caused by the inaccuracy of the fixed position of antenna onboard the satellite. This error is a random error for multiple repeated satellite missions. But for a single launch, it is considered to be a fixed bias vector in satellite body coordinate system during satellite flying.

The fixing errors of GPS receiver antenna in body coordinate system of two satellites are assumed as follows:

$$
\begin{aligned}
& \Delta \mathbf{E}_{1}=\left(\Delta x_{1} ; \Delta y_{1} ; \Delta z_{1}\right), \\
& \Delta \mathbf{E}_{2}=\left(\Delta x_{2} ; \Delta y_{2} ; \Delta z_{2}\right) .
\end{aligned}
$$


For a mutually observed GPS satellite $j$, the LOS vectors of two formation satellites are assumed to be $\mathbf{e}_{1}^{j}$ and $\mathbf{e}_{2}^{j}$. The impact of fixing errors of GPS receiver antenna for both formation satellites on GPS observation data can be denoted as

$$
\begin{aligned}
& \Delta \delta_{1}^{j}=\left(\mathbf{e}_{1}^{j}\right)^{T} \cdot \mathbf{M}_{\text {Body_CIRF, } 1} \cdot \Delta \mathbf{E}_{1}, \\
& \Delta \delta_{2}^{j}=\left(\mathbf{e}_{2}^{\mathbf{j}}\right)^{T} \cdot \mathbf{M}_{\text {Body_CIRF, } 2} \cdot \Delta \mathbf{E}_{2} .
\end{aligned}
$$

The impact of fixing error of GPS receiver antenna on differential GPS observation is

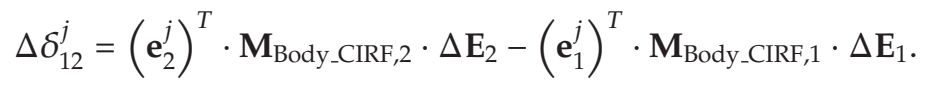

Due to the close separation of two satellites, we can assume

$$
\mathbf{e}_{1}^{j} \approx \mathbf{e}_{2}^{j}
$$

From (3.16) and (3.17), we can get

$$
\begin{aligned}
\Delta \delta_{12}^{\mathrm{j}} & \approx\left(\mathbf{e}_{2}^{j}\right)^{T} \cdot\left(\mathbf{M}_{\text {Body_CIRF }, 2} \cdot \Delta \mathbf{E}_{2}-\mathbf{M}_{\text {Body_CIRF }, 1} \cdot \Delta \mathbf{E}_{1}\right) \\
& =\left(\mathbf{e}_{2}^{j}\right)^{T} \cdot \mathbf{M}_{\text {Body_CIRF }, 2} \cdot\left(\Delta \mathbf{E}_{2}-\Delta \mathbf{E}_{1}\right)+\left(\mathbf{e}_{2}^{j}\right)^{T} \cdot\left(\mathbf{M}_{\text {Body_CIRF }, 2}-\mathbf{M}_{\text {Body_CIRF }, 1}\right) \cdot \Delta \mathbf{E}_{1} .
\end{aligned}
$$

As the magnitudes of $\Delta \mathbf{E}_{1}$ and $\Delta \mathbf{E}_{2}$ are small (generally less than $0.5 \mathrm{~mm}$ ) and the difference between $\mathbf{M}_{\text {Body_CIRF,1 }}$ and $\mathbf{M}_{\text {Body_CIRF,2 }}$ is insignificant; therefore, the impact of $\left(\mathbf{e}_{2}^{j}\right)^{T}$. $\left(\mathbf{M}_{\text {Body_CIRF,2 }}-\mathbf{M}_{\text {Body_CIRF,1) }}\right) \cdot \Delta \mathbf{E}_{1}$ in (3.18) can be neglected and the main influence is from $\left(\mathbf{e}_{2}^{j}\right)^{T} \cdot \mathbf{M}_{\text {Body_CIRF, } 2} \cdot\left(\Delta \mathbf{E}_{2}-\Delta \mathbf{E}_{1}\right)$. If the magnitude of GPS receiver antenna fixing error is $0.5 \mathrm{~mm}$ for each formation satellite, the maximum 3-dimensional impact on relative positioning can reach to $1 \mathrm{~mm}$.

In addition, we can also draw a conclusion from the aforementioned analysis that the GPS receiver antenna bias caused by thermal distortions of satellites can be cancelled out by differential GPS observation.

\subsection{Errors Related to Baseline Transformation}

From (2.1), errors related to baseline transformation consist of two parts: one part is introduced by transformation matrices $\mathbf{M}_{1}$ and $\mathbf{M}_{2}$, which is mainly caused by the satellite attitude measurement error; the other part is introduced by $\mathbf{S}_{1} \mathbf{O}_{1}$ and $\mathbf{S}_{2} \mathbf{O}_{2}$, which is caused by the inconsistency of two SAR antenna phase centers. 


\subsubsection{Error of Satellite Attitude Measurement}

Take $\mathbf{M}_{1}$ for instance,

$$
\mathbf{M}_{1}=\mathbf{M}_{\text {CIRF_ITRF }} \cdot \mathbf{M}_{\text {Body_CIRF }}
$$

where $\mathbf{M}_{\text {CIRF_ITRF }}$ is a transformation matrix from CIRF to ITRF, $\mathbf{M}_{\text {Body_ITRF }}$ has been defined in (3.2).

Note that the transformation from CIRF to ITRF is in accordance with IERS 1996 conventions [22] and this transformation error can be neglected. The errors of $\mathbf{M}_{1}$ and $\mathbf{M}_{2}$ are also introduced by satellite attitude measurement errors. Similar to the analysis of satellite attitude measurement error related to GPS measurement, from (3.8), we can obtain

$$
\sigma_{\mathbf{M}_{1} \cdot \mathbf{S}_{1} \mathbf{O}_{1}}^{2} \leq\left|\mathbf{S}_{1} \mathbf{O}_{1}\right|^{2} \cdot\left[\operatorname{Var}\left(\varepsilon_{\varphi}\right)+\left(\mathbf{E}\left(\varepsilon_{\varphi}\right)\right)^{2}+\operatorname{Var}\left(\varepsilon_{\theta}\right)+\left(\mathbf{E}\left(\varepsilon_{\theta}\right)\right)^{2}+\operatorname{Var}\left(\varepsilon_{\psi}\right)+\left(\mathbf{E}\left(\varepsilon_{\psi}\right)\right)^{2}\right] .
$$

Hence, the impact of attitude determination errors on baseline transformation is given as follows:

$$
\sigma_{\mathrm{Att}} \leq \sqrt{\sigma_{\mathbf{M}_{1} \cdot \mathbf{S}_{1} \mathbf{O}_{1}}^{2}+\sigma_{\mathbf{M}_{2} \cdot \mathbf{S}_{2} \mathbf{O}_{2}}^{2}}
$$

Take the attitude determination accuracy of TanDEM-X mission for instance and select the magnitudes of $\mathbf{S}_{1} \mathbf{O}_{1}$ and $\mathbf{S}_{2} \mathbf{O}_{2}$ as follows

$$
\left|\mathbf{S}_{1} \mathbf{O}_{1}\right|=\left|\mathbf{S}_{2} \mathbf{O}_{2}\right|=2 \mathrm{~m},
$$

we can get

$$
\sigma_{\mathrm{Att}} \leq 0.50 \mathrm{~mm} \text {. }
$$

\subsubsection{Consistency Error of SAR Antenna Phase Center}

Unlike GPS receiver antenna, active phased array antenna is selected for SAR antenna. The phase center of the SAR antenna describes the variation of the phase curve within the coverage region against a defined origin, here the origin of the antenna coordinate system [18]. For two formation satellites of InSAR mission, the same type of SAR antenna should be selected. As the identical processes of the scheme designing, manufacturing, and testing are selected for SAR antennas of the same type, theoretically the consistency in configuration and electric performance of SAR antennas should be well achieved. But factually there exist the errors during manufacturing, fixing, and deploying of SAR antenna, therefore, the consistency error of the SAR antenna phase center corresponding to the same beam occurs. It is mainly caused by two factors:

(1) The inconsistency between receiver channels, which is introduced by manufacturing process, such as the instrument difference, machining art level, module assembling level and the work temperature difference, et al. 
Table 1: Orbit elements of formation satellites.

\begin{tabular}{lcc}
\hline Parameters of satellites & Satellite 1 & Satellite 2 \\
\hline Semimajor axis & $6886478 \mathrm{~m}$ & $6886478 \mathrm{~m}$ \\
Inclination & $97.4438^{\circ}$ & $97.4438^{\circ}$ \\
Eccentricity & 0.00117 & 0.001073 \\
Argument of perigee & $90^{\circ}$ & $90^{\circ}$ \\
Right ascension of ascending node & $0^{\circ}$ & $0.01171^{\circ}$ \\
True anomaly & $269.99677^{\circ}$ & $270.00622^{\circ}$ \\
\hline
\end{tabular}

(2) The inconsistency between the locations of apertures, which is mainly caused by the fixing flatness difference, relative dislocation difference, the deployment inconsistency of SAR antennas and the configuration distortions caused by different thermal circumstances, and others.

According to current ability of engineering, the phase inconsistency between $T / R$ modules at X-band can be constrained to $15^{\circ}(3 \sigma)$ and the inconsistency between the locations of apertures can be constrained to $\lambda / 10(3 \sigma)$ [23] that equals to $36^{\circ}(3 \sigma)$ of phase inconsistency. Assuming that the number of T/R modules of an SAR antenna is $N$, the synthetic phase consistency error can be constrained to $\sqrt{\left(\left(15^{\circ}\right)^{2}+\left(36^{\circ}\right)^{2}\right) / N}=$ $39^{\circ} / \sqrt{N}(3 \sigma)$. Hence, the consistency error of two SAR antenna phase center locations can be constrained to

$$
\frac{\left(39^{\circ} / \sqrt{N}\right)}{360^{\circ}} \cdot \sqrt{2} \cdot \lambda=0.153 \cdot \frac{\lambda}{\sqrt{N}}(3 \sigma) .
$$

Take the TanDEM-X mission, for example. Setting $N=384, \lambda=0.032 \mathrm{~m}$, the consistency error of SAR antenna phase center location can be constrained to $0.25 \mathrm{~mm}(3 \sigma)$.

\section{Simulations for InSAR Spatial Baseline Determination}

\subsection{Simulation Settings}

The HELIX satellite formation is selected for the simulations and the orbit elements of two satellites are shown in Table 1 . The spaceborne SAR is assumed to work at X-band with a wavelength of $0.032 \mathrm{~m}$ and consist of $384 \mathrm{~T} / \mathrm{R}$ modules.

The entire simulation consists of GPS measurement simulation and baseline transformation simulation. The flow chart of GPS observation data simulation is shown in Figure 5. The International Reference Ionosphere 2007 (IRI2007) model is used to simulate ionospheric delay, Allan variation is used to simulate the clock offset of GPS receiver, and the ARP data, PCO data [18] and PCVs data of GPS receiver antenna system SEN67-1575-14+CRG are used to simulate the GPS receiver antenna phase center locations. The PCVs data contains the mean values and RMS values corresponding to frequency, azimuth, and elevation of received signal. The attitude data of formation satellite is generated as follows: at first, a transformation matrix from CIRF to satellite orbit coordinate system is obtained from orbit data of a formation satellite in CIRF; second, assuming the real Euler angles are $0^{\circ}$, that is, satellite orbit coordinate system and satellite body coordinate system are the same, the 


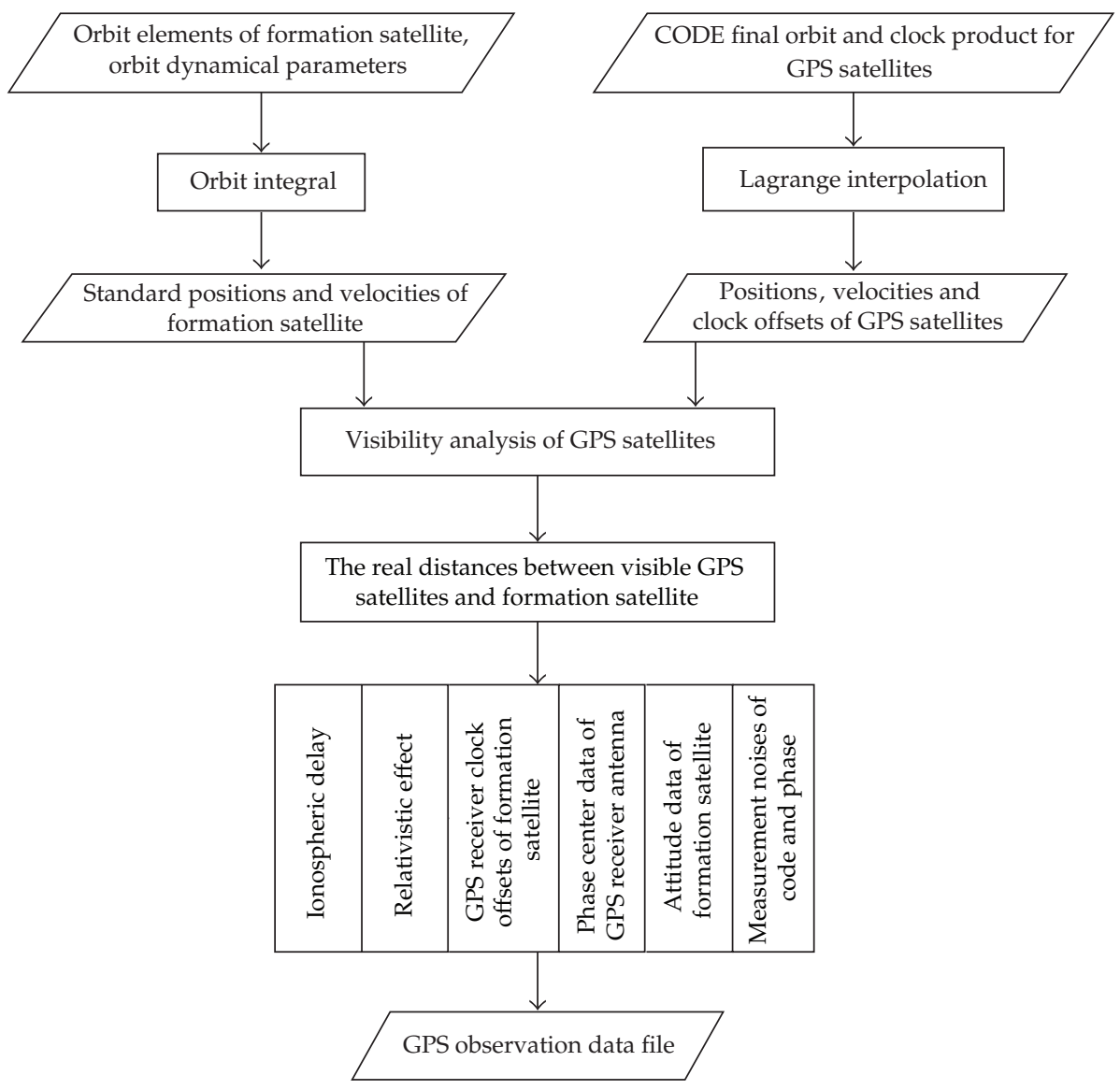

Figure 5: Flow chart of GPS observation data simulation.

simulating data of Euler angles are generated by attitude measurement error model list in Table 2; third, the transformation matrix from satellite orbit coordinate system to satellite body coordinate system can be obtained by the simulating data of Euler angles; at last, the attitude quaternion is obtained by the transformation matrix from CIRF to satellite body coordinate system.

Baseline transformation simulation is the process that the spatial baseline in ITRF is obtained by mass center data of formation satellites in ITRF, attitude simulation data, and SAR antenna phase center simulation locations in satellite body coordinate system. The real SAR antenna phase center simulation location in satellite body coordinate system is $(1.2278 \mathrm{~m}, 1.5876 \mathrm{~m}, 0.0223 \mathrm{~m})$. The error accuracies and models in the simulations are shown in Table 2.

\subsection{Simulations of Errors Related to GPS Measurement}

Each error related to GPS measurement is analyzed by single factor simulation, which is intended to obtain its impact on relative positioning based on dual-frequency GPS. The impact of each error is drawn by the comparison residuals between the relative position 
Table 2: Error accuracies and modeling descriptions in simulations.

\begin{tabular}{|c|c|c|}
\hline Error type & Error accuracy & Modeling description \\
\hline $\begin{array}{l}\text { GPS code measurement } \\
\text { noise }\end{array}$ & $0.5 \mathrm{~m}(1 \sigma)$ & $\begin{array}{l}\text { Gaussian white noise model with mean value of } \\
0 \mathrm{~m} \text { and standard deviation of } 0.5 \mathrm{~m}\end{array}$ \\
\hline $\begin{array}{l}\text { GPS carrier phase } \\
\text { measurement noise }\end{array}$ & $0.002 \mathrm{~m}(1 \sigma)$ & $\begin{array}{l}\text { AR(2) model with mean value of } 0 \mathrm{~m} \text { and standard } \\
\text { deviation of } 0.002 \mathrm{~m}\end{array}$ \\
\hline $\begin{array}{l}\text { Ground calibration error } \\
\text { of GPS receiver antenna } \\
\text { phase center }\end{array}$ & - & $\begin{array}{l}\text { ARP data, PCO data, and PCVs data (mean value } \\
\text { data and RMS data) of SEN67-1575-14+CRG }\end{array}$ \\
\hline $\begin{array}{l}\text { Attitude measurement } \\
\text { error }\end{array}$ & $\begin{array}{l}\text { Fixed bias of } 0.005^{\circ} \\
\text { in the yaw, pitch, } \\
\text { and roll components } \\
\text { plus a } 0.003^{\circ}(1 \sigma) \\
\text { random error }\end{array}$ & $\begin{array}{l}\text { Gaussian white noise model with mean value of } \\
0.005^{\circ} \text { and standard deviation of } 0.003^{\circ} \text { in the yaw, } \\
\text { pitch, and roll components }\end{array}$ \\
\hline $\begin{array}{l}\text { Fixing error of GPS } \\
\text { receiver antenna }\end{array}$ & $0.5 \mathrm{~mm}(3 \sigma)$ & $\begin{array}{l}\text { A fixed vector with direction randomly drawn in } \\
\text { unit ball and magnitude of } 0.5 \mathrm{~mm} \text { in each satellite } \\
\text { body coordinate system }\end{array}$ \\
\hline $\begin{array}{l}\text { Consistency error of SAR } \\
\text { antenna phase center }\end{array}$ & $0.25 \mathrm{~mm}(3 \sigma)$ & $\begin{array}{l}\text { A fixed vector with direction randomly drawn in } \\
\text { unit ball and magnitude of } 0.25 \mathrm{~mm} \text { in body } \\
\text { coordinate system of Satellite } 1\end{array}$ \\
\hline
\end{tabular}

solutions determined by GPS observation data and relative positions obtained by standard orbits of formation satellites. The relative position solutions are implemented in the separate software tools as part of the NUDT Orbit Determination Software 1.0. The GPS observation data processing consists of GPS observation data preprocessing [24], reduced dynamic precise orbit determination for single satellite [25], GPS observation data editing [17, 24], and reduced dynamic precise relative positioning. The RMS values of KBR comparison residuals of GRACE relative position solution are about 1-2 $\mathrm{mm}$ implemented by this software.

\subsubsection{Simulations for GPS Carrier Phase Measurement Noise}

The noises of GPS carrier phase ( $L 1$ and $L 2)$ measurements are separately simulated by second-order autoregressive model $(\mathrm{AR}(2))$ as follows

$$
e_{L}^{j}\left(t_{i}\right)=e_{L}^{j}\left(t_{i-1}\right)-0.67 \cdot e_{L}^{j}\left(t_{i-2}\right)+\varepsilon_{L}^{j}\left(t_{i}\right)
$$

where $\varepsilon_{L}^{j}\left(t_{i}\right)$ is the noise of carrier phase $L$ measurement for GPS satellite $j$ at epoch $t_{i}, \varepsilon_{L}^{j}\left(t_{i}\right)$ is the Gaussian white noise.

From the following formula

$$
\mathbf{E}\left(e_{L}^{j}\left(t_{i}\right)\right)=0 \mathrm{~m} ; \quad \sigma\left(e_{L}^{j}\left(t_{i}\right)\right)=0.002 \mathrm{~m},
$$

where $\sigma(\cdot)$ denotes the standard deviation of a random variable, we can get

$$
\mathbf{E}\left(\varepsilon_{L}^{j}\left(t_{i}\right)\right)=0 \mathrm{~m} ; \quad \sigma\left(\varepsilon_{L}^{j}\left(t_{i}\right)\right)=0.0012 \mathrm{~m} .
$$

One instance of carrier phase noise simulation is shown in Figure 6. 


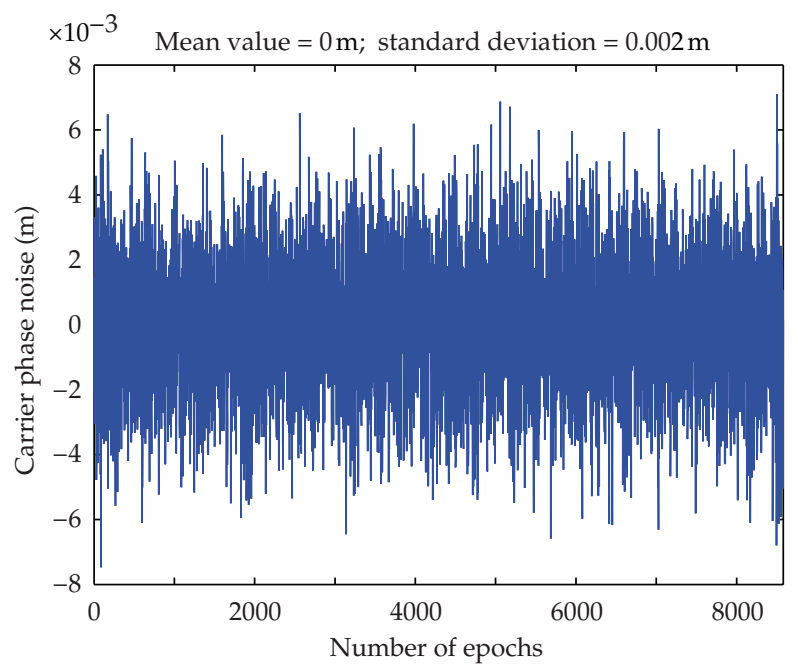

Figure 6: One instance of carrier phase measurement noise simulation.

50 groups of $24 \mathrm{~h}$ GPS observation data (interval of $30 \mathrm{~s}$ ) for two formation satellites are simulated by only adding the noises of GPS carrier phase ( $L 1$ and $L 2$ ) measurements. By the processing of relative positioning, the mean RMS values of comparison residuals of relative position solutions in ITRF (Figure 7) are $0.340 \mathrm{~mm}$ of $x$-axis, $0.333 \mathrm{~mm}$ of $y$-axis, $0.288 \mathrm{~mm}$ of $z$-axis, and $0.560 \mathrm{~mm}$ of 3 dimensions. It is shown by simulation results that the GPS carrier phase noise can be well smoothed by reduced dynamic relative positioning approach.

\subsubsection{Simulations for Ground Calibration Error of GPS Receiver Antenna Phase Center}

Ground calibration error of GPS receiver antenna phase center is mainly caused by PCVs. The PCVs values are described by the mean value and RMS value corresponding to the direction of received signal. The PCV value corresponding to the direction of received signal is simulated by Gaussian white noise with mean value and RMS value obtained from ground calibration result of GPS receiver antenna system SEN67-1575-14+CRG.

The GPS observation data are simulated only considered ground calibration error of GPS receiver antenna phase center. By the precise orbit determination for single satellite, the mean RMS values of comparison residuals of orbit solutions in ITRF are $4.018 \mathrm{~mm}$ of $x$-axis, $4.154 \mathrm{~mm}$ of $y$-axis, $2.427 \mathrm{~mm}$ of $z$-axis, and $6.269 \mathrm{~mm}$ of 3 dimensions. The impacts of PCVs on single satellite orbit solutions are mainly made by the mean value part of PCVs, while the impacts of RMS part in ITRF are only $0.119 \mathrm{~mm}$ of $x$-axis, $0.094 \mathrm{~mm}$ of $y$-axis, $0.116 \mathrm{~mm}$ of $z$-axis, and $0.191 \mathrm{~mm}$ of 3 dimensions, and the RMS part of PCVs can nearly be smoothed. By the processing of relative positioning, the mean RMS values of comparison residuals of relative position solutions in ITRF are $0.067 \mathrm{~mm}$ of $x$-axis, $0.070 \mathrm{~mm}$ of $y$-axis, $0.056 \mathrm{~mm}$ of $z$ axis, and $0.112 \mathrm{~mm}$ of 3-dimensions. As the nearly equal models of ground calibration errors of GPS receiver antenna phase centers for two formation satellites are selected and the LOS vectors are nearly the same for close satellite formation, the impacts of mean value part of PCVs can nearly be cancelled out by differential GPS observation and impacts of RMS part can be well smoothed by the constraints of orbit dynamical models. It is shown by the results 


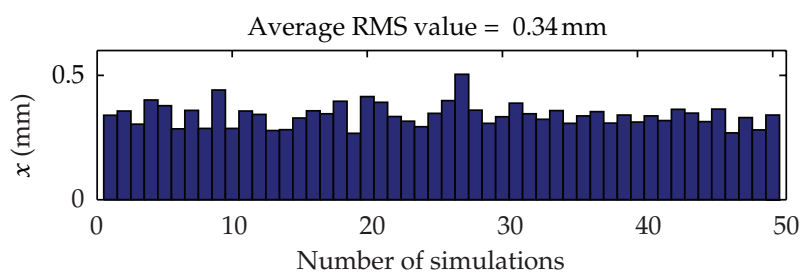

(a)

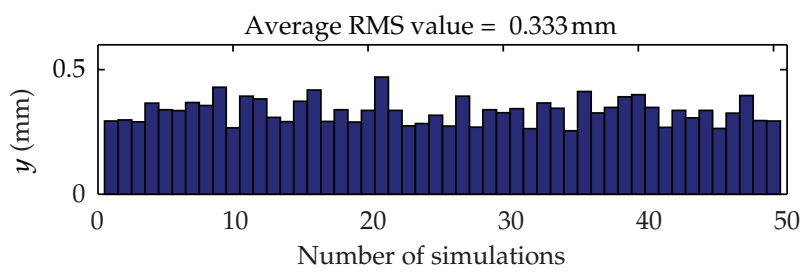

(b)

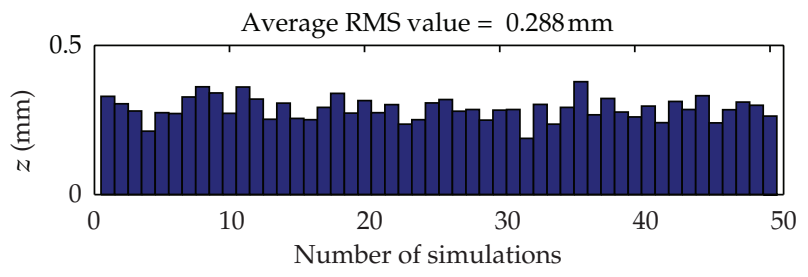

(c)

Figure 7: Simulation results of GPS carrier phase measurement noise for relative positioning.

of single satellite orbit solutions and relative position solutions that the characters of GPS receiver antenna phase centers onboard two formation satellites must have great consistency.

\subsubsection{Simulations of Satellite Attitude Measurement Error for GPS Relative Positioning}

The Euler angle errors are simulated by Gaussian white noise with $0.005^{\circ}$ of mean value and $0.003^{\circ}$ of standard deviation, and 50 groups of $24 \mathrm{~h}$ GPS observation data for two formation satellites are simulated by only adding the attitude measurement errors. The mean RMS values of comparison residuals of relative position solutions in ITRF (Figure 8 ) are $0.069 \mathrm{~mm}$ of $x$-axis, $0.075 \mathrm{~mm}$ of $y$-axis, $0.081 \mathrm{~mm}$ of $z$-axis, and $0.128 \mathrm{~mm}$ of 3 dimensions. The 3 dimensional maximum of comparison residuals in these 50 simulations is $0.219 \mathrm{~mm}$, which is less than $0.47 \mathrm{~mm}$ and is well consistent with aforementioned analysis in Section 3.1.3.

\subsubsection{Simulations for Fixing Error of GPS Receiver Antenna}

The fixing error of GPS receiver antenna belongs to systematic error and it is a fixed bias vector in satellite body coordinate system. At first, four representatively "extreme" circumstances of fixing errors of GPS receiver antennas onboard two formation satellites are simulated. The so-called "extreme" circumstance is that the directions of two fixed bias vectors are opposite. Four representatively "extreme" circumstances of fixing errors of GPS receiver antennas here are directions along $X$-axis, $Y$-axis, $Z$-axis, and diagonal of $X$-axis, 


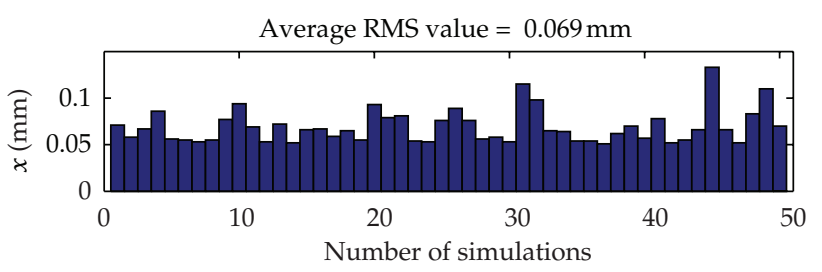

(a)

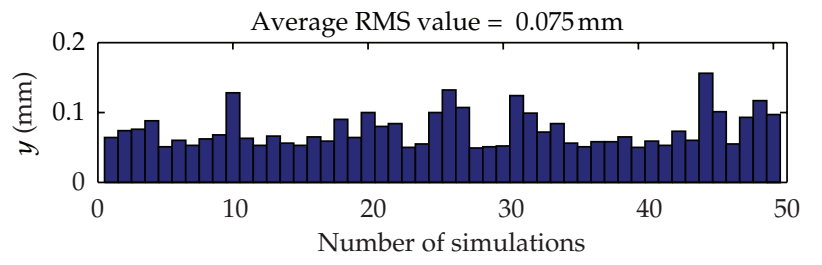

(b)

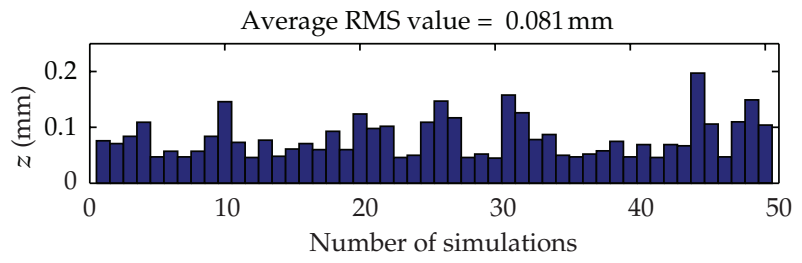

(c)

Figure 8: Simulation results of satellite attitude measurement error for relative positioning.

Table 3: Relative positioning results for four representatively "extreme" circumstances of fixing errors of GPS receiver antenna.

\begin{tabular}{lcccc}
\hline & $x / \mathrm{mm}$ & $y / \mathrm{mm}$ & $z / \mathrm{mm}$ & 3 dimension $/ \mathrm{mm}$ \\
\hline$X$-axis & 0.512 & 0.499 & 0.701 & 1.002 \\
$Y$-axis & 0.677 & 0.694 & 0.133 & 0.979 \\
Z-axis & 0.072 & 0.083 & 0.081 & 0.136 \\
Diagonal & 0.502 & 0.503 & 0.429 & 0.830 \\
\hline
\end{tabular}

$Y$-axis, Z-axis in satellite body coordinate system, respectively. All the magnitudes of fixed bias vectors are selected $0.5 \mathrm{~mm}$. $24 \mathrm{~h}$ GPS observation data for two formation satellites are simulated by only considering the four representatively "extreme" circumstances of fixing errors of GPS receiver antennas. The results of relative positioning are shown in Table 3.

From Table 3, it is shown that the fixing errors of GPS receiver antenna along X-axis and $Y$-axis will mainly be absorbed by relative position solutions and the impact can reach to $1 \mathrm{~mm}$, but the error along $Z$-axis can be smoothed by the constraints of orbit dynamical models.

In practice, the occurrence of "extreme" circumstances is extremely low and they are just analyzed as the ultimate circumstances. For multiple repeated satellite missions, the fixing error of GPS receiver antenna is a random error. So this error can be simulated as a fixed vector with direction randomly drawn from unit ball and magnitude of $0.5 \mathrm{~mm}$ in each satellite body coordinate system. 50 groups of $24 \mathrm{~h}$ GPS observation data for two formation satellites are simulated by only adding the simulations of fixing error of GPS receiver 


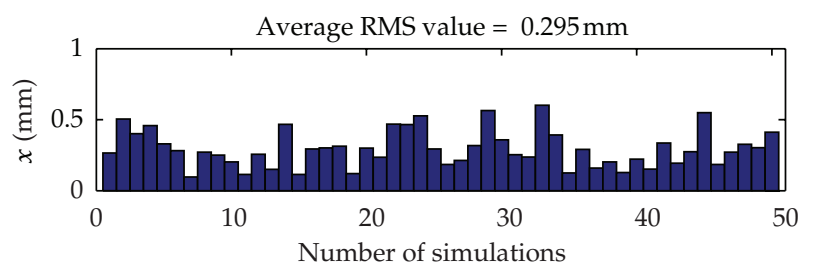

(a)

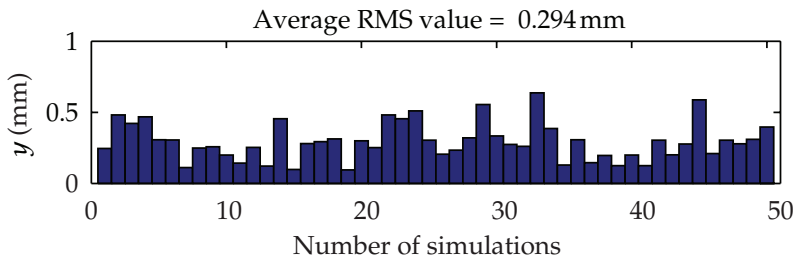

(b)

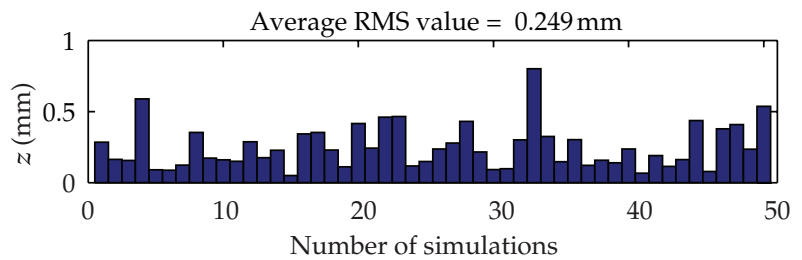

(c)

Figure 9: Simulation results of fixing error of GPS receiver antenna for relative positioning.

antenna. The mean RMS values of comparison residuals of relative position solutions in ITRF (Figure 9) are $0.295 \mathrm{~mm}$ of $x$-axis, $0.294 \mathrm{~mm}$ of $y$-axis, $0.249 \mathrm{~mm}$ of $z$-axis, and $0.495 \mathrm{~mm}$ of 3 dimensions.

From aforementioned simulations of each error related to GPS measurement, it is shown that the impacts of GPS carrier phase measurement noise and fixing error of GPS receiver antenna on GPS relative positioning are much bigger than other errors related to GPS measurement and these two errors are the main factors of errors related to GPS measurement.

\subsection{Simulations of Errors Related to Baseline Transformation}

In this section, the impact of each error on baseline transformation is obtained by single factor simulation. Each impact is given by the comparison between the spatial baseline solutions obtained with and without errors.

\subsubsection{Simulations of Satellite Attitude Measurement Error for Baseline Transformation}

The satellite attitude simulation data used here are the same as Section 4.2.3. By baseline transformation with attitude simulation data, the mean RMS values of comparison residuals of spatial baseline solutions in ITRF (Figure 10) are $0.115 \mathrm{~mm}$ of $x$-axis, $0.115 \mathrm{~mm}$ of $y$ axis, $0.133 \mathrm{~mm}$ of $z$-axis, and $0.210 \mathrm{~mm}$ of 3 dimensions. The 3 dimensional maximum of 


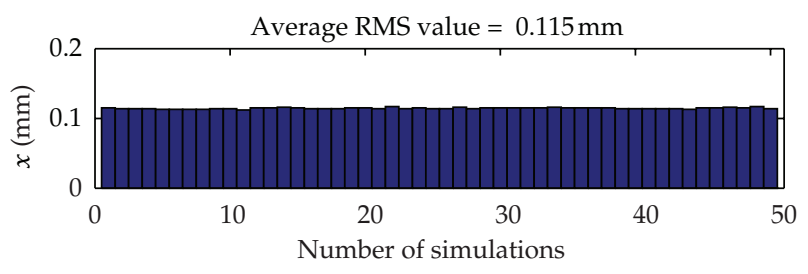

(a)

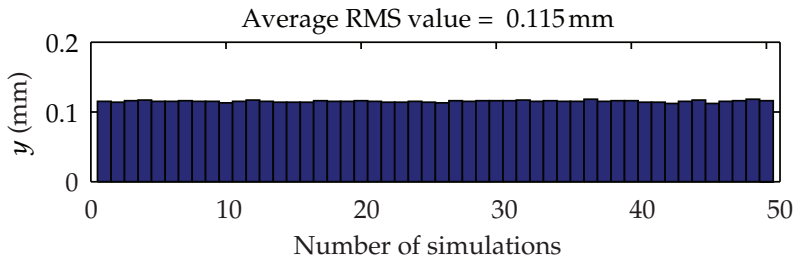

(b)

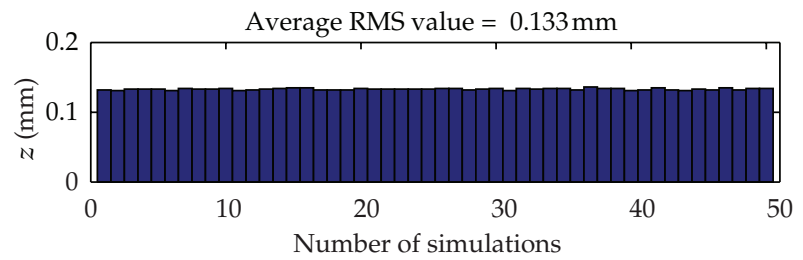

(c)

Figure 10: Simulation results of satellite attitude measurement error for baseline transformation.

comparison residuals in these 50 simulations is $0.213 \mathrm{~mm}$, which is less than $0.50 \mathrm{~mm}$ and is consistent with aforementioned analysis in Section 3.2.1.

\subsubsection{Simulations for Consistency Error of SAR Antenna Phase Center}

It is shown by the analysis in Section 3.2.2 that the accuracy of consistency error of SAR antenna phase center is better than $0.25 \mathrm{~mm}(3 \sigma)$ in current simulation circumstances. This error is only added to the SAR antenna phase center of Satellite 1 and can be simulated as a fixed vector with direction randomly drawn from unit ball and magnitude of $0.25 \mathrm{~mm}$ in body coordinate system of satellite 1 . By 50 groups of simulations, the mean RMS values of comparison residuals of spatial baseline solutions in ITRF (Figure 11) are $0.142 \mathrm{~mm}$ of $x$-axis, $0.142 \mathrm{~mm}$ of $y$-axis, and $0.153 \mathrm{~mm}$ of $z$-axis.

\subsection{Simulations of Total Error Sources}

In this section, all the errors are added to the flow of spatial baseline determination simulations according to the error models listed in Table 2. By 50 groups of total error sources simulations, the mean RMS values of comparison residuals of spatial baseline solutions in ITRF (Figure 12) are $0.500 \mathrm{~mm}$ of $x$-axis, $0.500 \mathrm{~mm}$ of $y$-axis, $0.452 \mathrm{~mm}$ of $z$-axis, and $0.845 \mathrm{~mm}$ of 3 dimensions. 


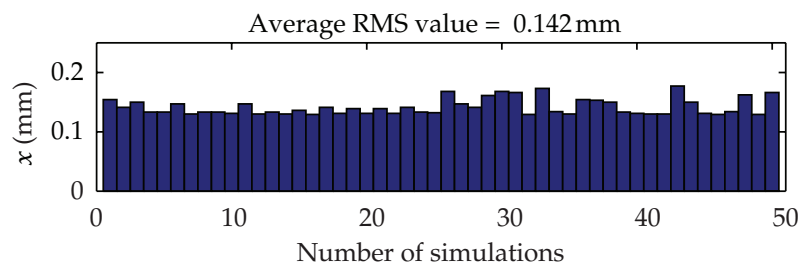

(a)

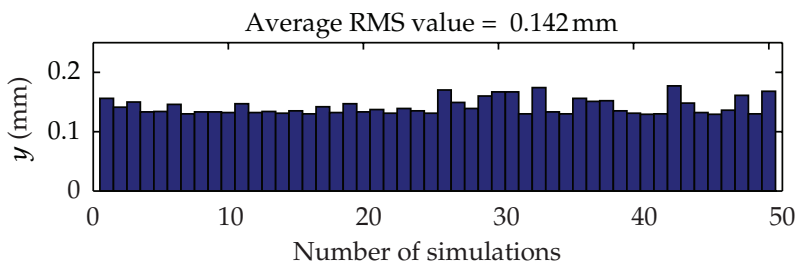

(b)

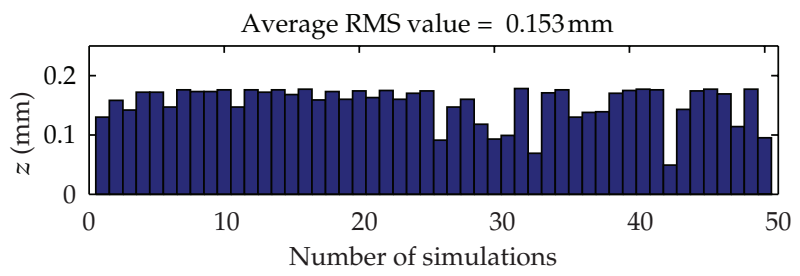

(c)

Figure 11: Simulation results of consistency error of SAR antenna phase center for baseline transformation.

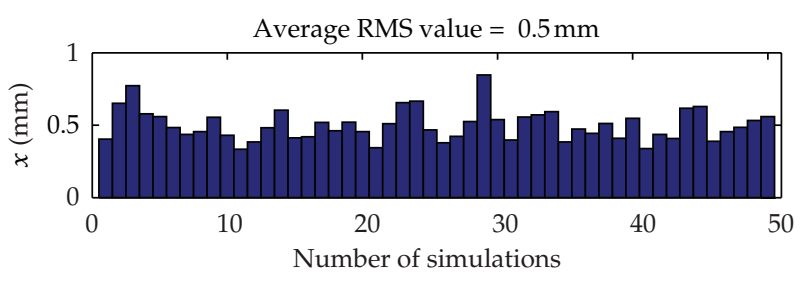

(a)

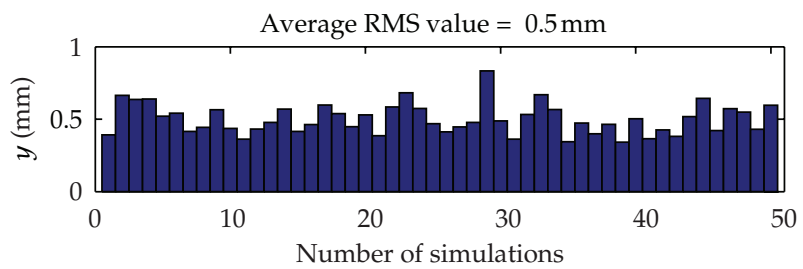

(b)

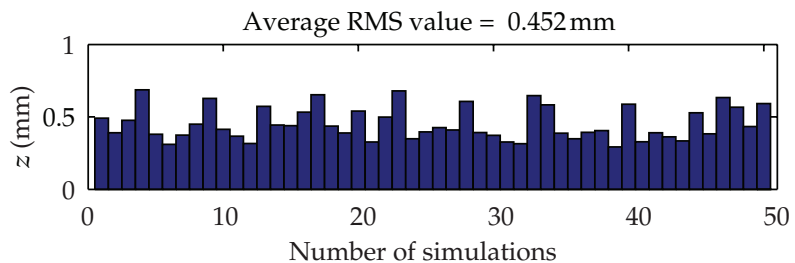

(c)

Figure 12: Simulation results of total error sources for spatial baseline determination. 


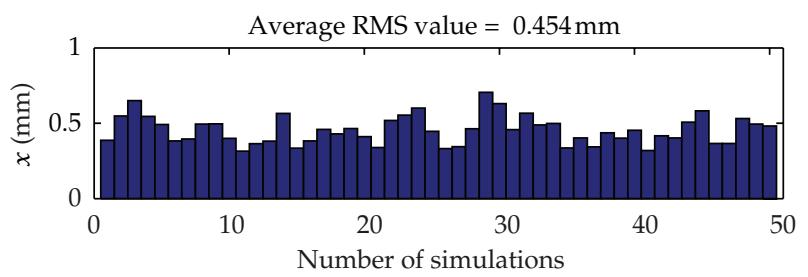

(a)

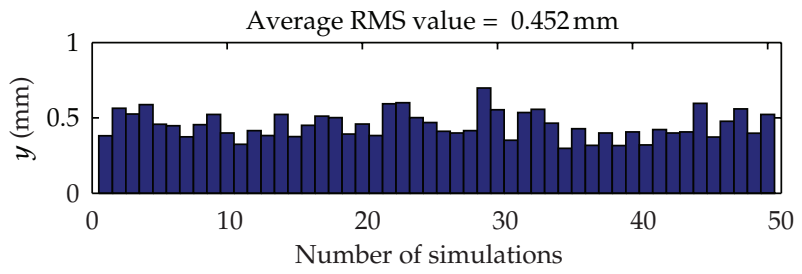

(b)

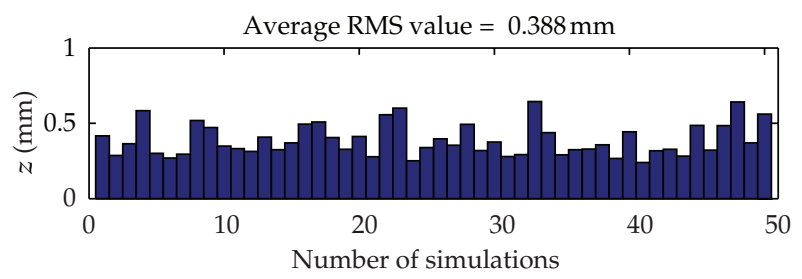

(c)

Figure 13: Simulation results of total errors related to GPS measurement for relative positioning.

In addition, the impact of total errors related to GPS measurement on GPS relative positioning in ITRF (Figure 13) is $0.454 \mathrm{~mm}$ of $x$-axis, $0.452 \mathrm{~mm}$ of $y$-axis, $0.388 \mathrm{~mm}$ of $z$ axis, and $0.755 \mathrm{~mm}$ of 3 dimensions, and the impact of total errors related to baseline transformation in ITRF (Figure 14) is $0.185 \mathrm{~mm}$ of $x$-axis, $0.185 \mathrm{~mm}$ of $y$-axis, $0.206 \mathrm{~mm}$ of $z$-axis, and $0.334 \mathrm{~mm}$ of 3 dimensions.

It is shown by the simulations of total error sources that errors related to GPS measurement are the main error sources for the spatial baseline determination and $1 \mathrm{~mm}$ level InSAR spatial baseline determination can be realized according to current simulation conditions.

\section{Conclusions}

In this paper, the errors introduced by spatial baseline measurement for InSAR mission are deeply studied. The impacts of errors on spatial baseline determination are analyzed by single factor simulations and total error sources simulations. The main conclusions are drawn as follows.

(1) The spatial baseline measurement errors can be classified into two groups: errors related to GPS measurement and errors related to baseline transformation. By simulations, the three-dimensional impacts of these errors on spatial baseline determination in ITRF are $0.755 \mathrm{~mm}$ and $0.334 \mathrm{~mm}$, respectively. It is shown that 


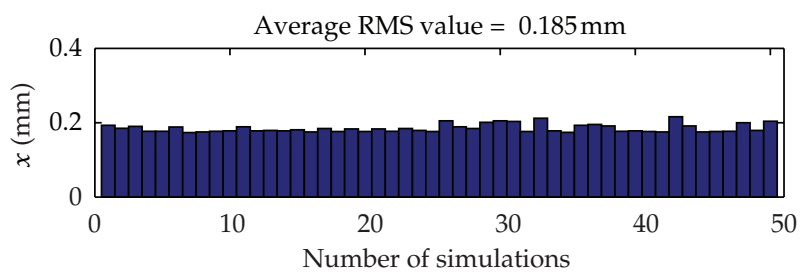

(a)

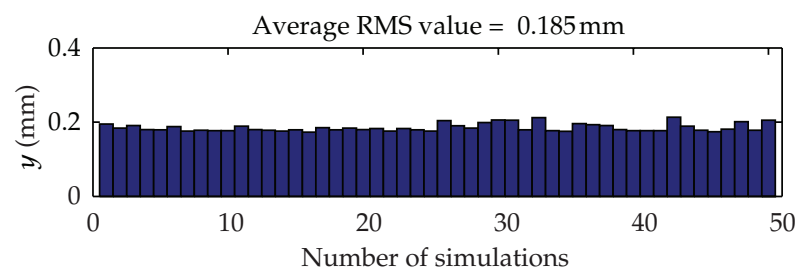

(b)

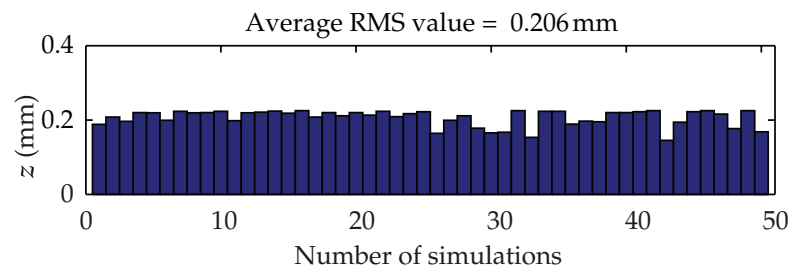

(c)

Figure 14: Simulation results of total errors related to baseline transformation for baseline transformation.

the errors related to GPS measurement are the main influence on spatial baseline determination.

(2) By the results of single factor simulations, the three dimensional impacts of GPS carrier phase measurement noise and the fixing error of GPS receiver antenna on GPS relative positioning in ITRF are $0.560 \mathrm{~mm}$ and $0.495 \mathrm{~mm}$, respectively. These two errors are the main factors of errors related to GPS measurement.

(3) It is shown by total error sources simulations that the impact of all the errors on spatial baseline determination in ITRF is $0.500 \mathrm{~mm}$ of $x$-axis, $0.500 \mathrm{~mm}$ of $y$-axis, $0.452 \mathrm{~mm}$ of $z$-axis, and $0.845 \mathrm{~mm}$ of 3 dimensions. Therefore, $1 \mathrm{~mm}$ level InSAR spatial baseline determination can be realized.

\section{Acknowledgments}

The mean antenna phase center description for the Sensor Systems SEN67157514 antenna has been contributed by the German Space Operations Center (GSOC), Deutsches Zentrum für Luft- und Raumfahrt (DLR), Wessling, to enable the simulation of antenna phase center data of dual-frequency GPS receiver. Precise GPS ephemerides for use within this study have been obtained from the Center for Orbit Determination in Europe at the Astronomical Institute of the University of Bern (AIUB). The authors extend special thanks to the support of the above institutions. This paper is supported by the National Natural Science Foundation of China 
(Grant no. 61002033 and no. 60902089) and Open Research Fund of State Key Laboratory of Astronautic Dynamics of China (Grant no. 2011ADL-DW0103).

\section{References}

[1] G. Krieger, I. Hajnsek, K. P. Papathanassiou, M. Younis, and A. Moreira, "Interferometric synthetic aperture radar (SAR) missions employing formation flying," Proceedings of the IEEE, vol. 98, no. 5, pp. 816-843, 2010.

[2] M. L. Jiao, "A review on latest Interferometric Synthetic Aperture Radar researches," in WRI World Congress on Software Engineering (WCSE '09), pp. 387-390, May 2009.

[3] W. Wang, "Optimal baseline design and error compensation for bistatic spaceborne InSAR," in Proceedings of Fringe Workshop, November-December 2005.

[4] G. Krieger, A. Moreira, H. Fiedler et al., "TanDEM-X: a satellite formation for high-resolution SAR interferometry," IEEE Transactions on Geoscience and Remote Sensing, vol. 45, no. 11, pp. 3317-3340, 2007.

[5] M. Zink, H. Fiedler, I. Hajnsek, G. Krieger, A. Moreira, and M. Werner, "The TanDEM-X mission concept," in IEEE International Geoscience and Remote Sensing Symposium (IGARSS '06), pp. 1938-1941, August 2006.

[6] R. Werninghaus and S. Buckreuss, "The TerraSAR-X mission and system design," IEEE Transactions on Geoscience and Remote Sensing, vol. 48, no. 2, pp. 606-614, 2010.

[7] M. Wermuth, O. Montenbruck, and A. Wendleder, "Relative navigation for the TanDEM-X mission and evaluation with DEM calibration results," in the 22nd International Symposium on Space Flight Dynamics, Sao Jose dos Campos, Brazil, 2011.

[8] H. Xu, Y. Zhou, and C. Li, "Analysis and simulation of spaceborne SAR interferometric baseline," in Proceedings of the CIE International Conference on Radar, pp. 639-643, Beijing, China, October 2001.

[9] R. Kroes, O. Montenbruck, W. Bertiger, and P. Visser, "Precise GRACE baseline determination using GPS," GPS Solutions, vol. 9, no. 1, pp. 21-31, 2005.

[10] O. Montenbruck, P. W. L. van Barneveld, Y. Yoon, and P. N. A. M. Visser, "GPS-based precision baseline reconstruction for the TanDEM-X SAR-formation," in the 20th International Symposium on Space Flight Dynamics, pp. 24-28, 2007.

[11] S. D'Amico and O. Montenbruck, "Differential GPS: an enabling technology for formation flying satellites," in the 7th IAA Symposium on Small Satellites for Earth Observation, pp. 457-464, 2009.

[12] O. Montenbruck and E. Gill, Satellite Orbits: Models, Methods and Applications, Springer, Heidelberg, Germany, 2000.

[13] P. W. Binning, Absolute and relative satellite to satellite navigation using GPS, Ph.D. dissertation, University of Colorado, 1997.

[14] P. J. G. Teunissen, "The least-squares ambiguity decorrelation adjustment: a method for fast GPS integer ambiguity estimation," Journal of Geodesy, vol. 70, no. 1-2, pp. 65-82, 1995.

[15] P. J. G. Teunissen, P. J. De Jonge, and C. C. J. M. Tiberius, “The least-squares ambiguity decorrelation adjustment: its performance on short GPS baselines and short observation spans," Journal of Geodesy, vol. 71, no. 10, pp. 589-602, 1997.

[16] J. Kouba, A guide using International GPS Service (IGS) products, Jet Propulsion Laboratory, Pasadena, Calif, USA, 2009.

[17] R. Kroes, Precise relative positioning of formation flying spacecraft using GPS, Ph.D. dissertation, Delft University of Technology, The Netherlands, 2006.

[18] O. Montenbruck, M. Garcia-Fernandez, Y. Yoon, S. Schön, and A. Jäggi, "Antenna phase center calibration for precise positioning of LEO satellites," GPS Solutions, vol. 13, no. 1, pp. 23-34, 2009.

[19] A. Jäggi, R. Dach, O. Montenbruck, U. Hugentobler, H. Bock, and G. Beutler, "Phase center modeling for LEO GPS receiver antennas and its impact on precise orbit determination," Journal of Geodesy, vol. 83, no. 12, pp. 1145-1162, 2009.

[20] M. Garcia and O. Montenbruck, "TerraSAR-X/TanDEM-X GPS antenna phase center analysis and results," German Space Operations Center, Germany, 2007.

[21] J. H. Gonzalez, M. Bachmann, G. Krieger, and H. Fiedler, "Development of the TanDEM-X calibration concept: analysis of systematic errors," IEEE Transactions on Geoscience and Remote Sensing, vol. 48, no. 2, pp. 716-726, 2010.

[22] D. D. McCarthy, "IERS conventions," IERS Technical Note 21, Observatoire de Paris, Paris, France, pp. 20-39, 1996. 
[23] W. T. Wang and Y. H. Qi, "A new technique to compensate for error in SAR antenna power pattern," Chinese Space Science and Technology, vol. 17, no. 3, pp. 65-70, 1997.

[24] D. F. Gu, The spatial states measurement and estimation of distributed InSAR satellite system, Ph.D. dissertation, National University of Defense Technology, China, 2009.

[25] O. Montenbruck, T. Van Helleputte, R. Kroes, and E. Gill, "Reduced dynamic orbit determination using GPS code and carrier measurements," Aerospace Science and Technology, vol. 9, no. 3, pp. 261$271,2005$. 


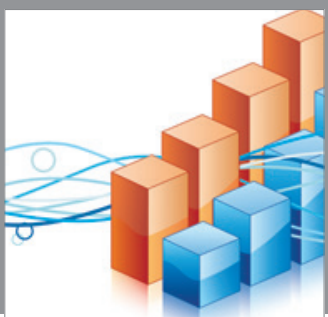

Advances in

Operations Research

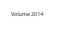

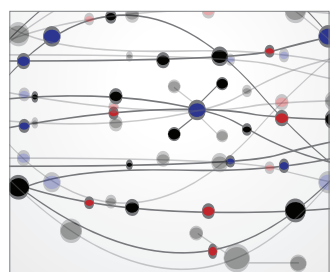

\section{The Scientific} World Journal
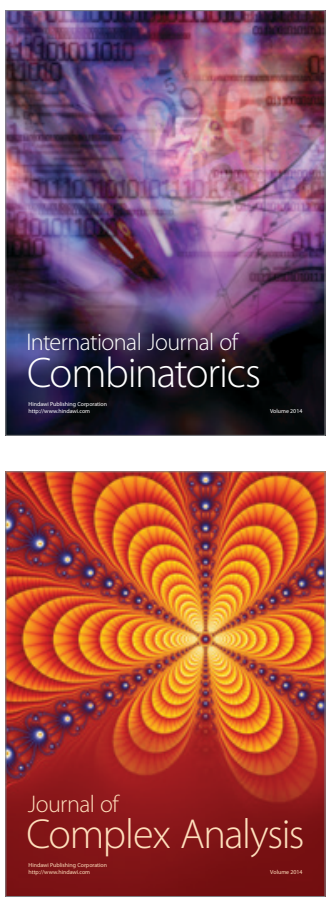

International Journal of

Mathematics and

Mathematical

Sciences
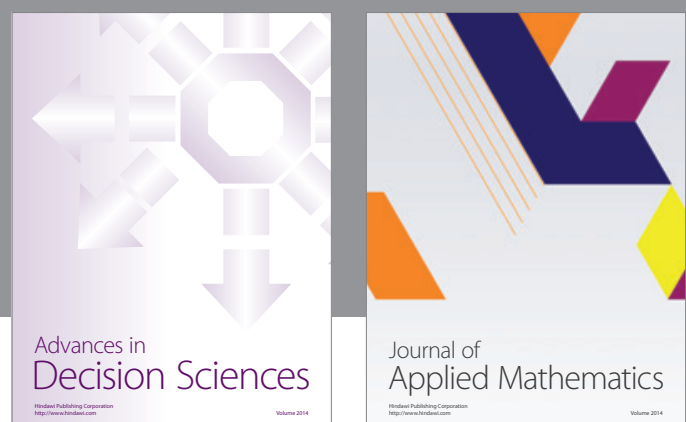

Journal of

Applied Mathematics
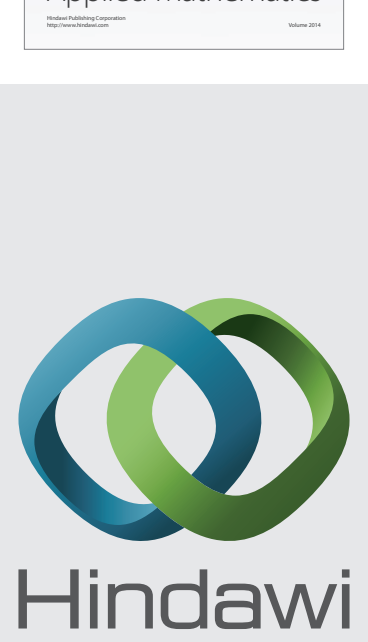

Submit your manuscripts at http://www.hindawi.com
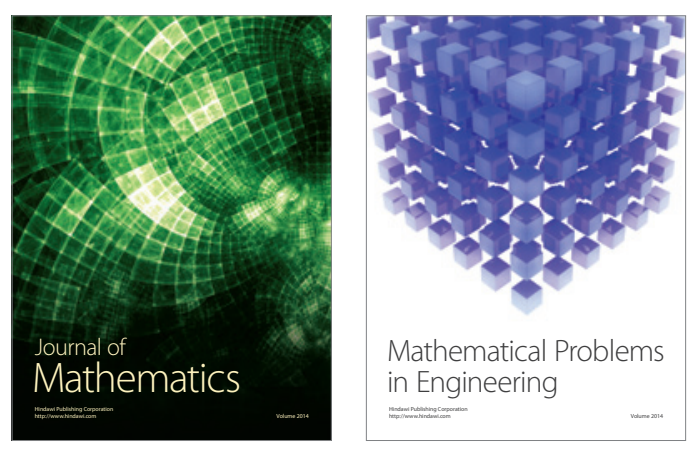

Mathematical Problems in Engineering
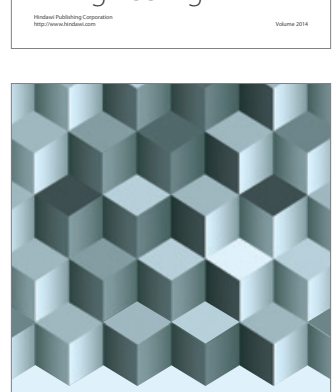

Journal of

Function Spaces
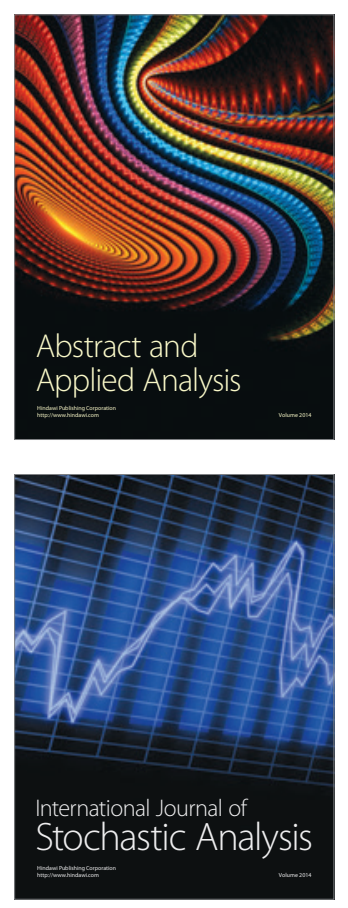

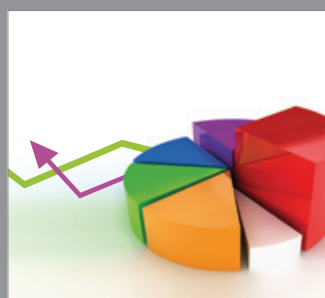

ournal of

Probability and Statistics

Promensencen
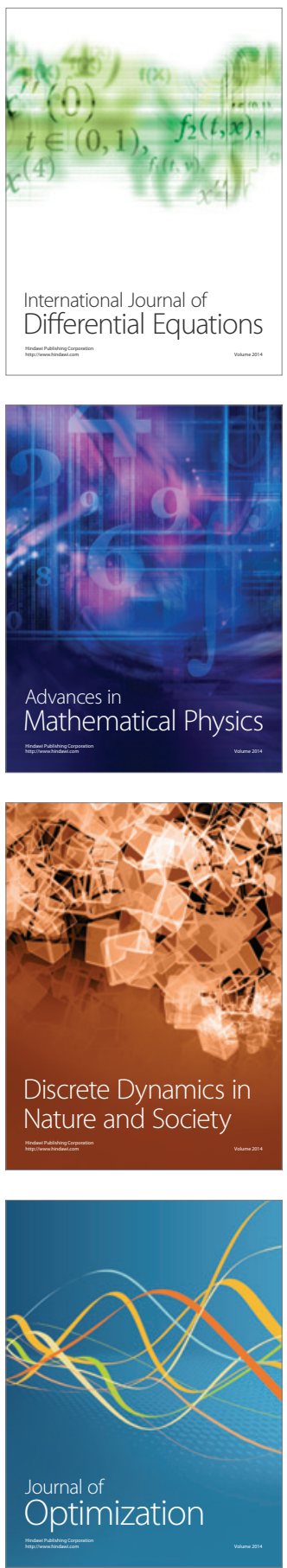